\title{
Distributed computing strategy for structural health monitoring
}

\author{
Y. Gao ${ }^{1}$, B. F. Spencer $\mathrm{Jr}^{2, *, \dagger, *}$ and M. Ruiz-Sandoval ${ }^{3, \S}$ \\ ${ }^{1}$ DMJM Harris Inc. 20 Exchange Place, New York, NY 10005, U.S.A. \\ ${ }^{2}$ Department of Civil and Environmental Engineering, University of Illinois at Urbana-Champaign, 205 N. Mathews Ave., \\ Urbana, IL 61801, U.S.A. \\ ${ }^{3}$ Universidad Autonoma Metropolitana-Azcapotzalco, Av. San Pablo No. 180, Col. Reynosa Tamaulipas, \\ C.P. 02200, México
}

\begin{abstract}
SUMMARY
Monitoring of complex structures to provide real-time safety and reliability information regarding the structure poses significant technical challenges. To detect damage in large civil infrastructure systems, densely distributed sensors are expected to be required. Use of traditional wired sensors is challenging for such applications because of the cost and difficulty in deploying and maintaining a large wiring plant. Using wireless sensor network is also difficult because large amounts of measured data need to be transferred to a central station. The bandwidth and power requirement to transfer these data may easily exceed the limit of the wireless sensor. Recently rapid advances in smart sensor technologies have made damage detection using a dense array of sensors feasible. The essential feature of a smart sensor is the on-board microprocessor, which allows smart sensors to make decisions, perform computation, save data locally, etc. By conducting a portion of the computation at the sensor level, only limited information needs to be transferred back to a central station. However, damage detection algorithms which can take advantage of the distributed computing environment offered by smart sensors are currently limited.

In this paper, a new distributed computing strategy for structural health monitoring is proposed that is suitable for implementation on a network of densely distributed smart sensors. In this approach, a hierarchical strategy is proposed in which adjacent smart sensors are grouped together to form sensor communities. A flexibility-based damage detection method is employed to evaluate the condition of the local elements within these communities by utilizing only locally measured information. The damage detection results in these communities are then communicated with the surrounding communities and sent back to a central station. Numerical simulation demonstrates that the proposed approach works well for both single and multiple damage scenarios. Copyright (C) 2005 John Wiley \& Sons, Ltd.
\end{abstract}

KEY WORDS: distributed computing strategy; structural health monitoring; flexibility matrix; smart sensors

\footnotetext{
*Correspondence to: B. F. Spencer Jr, Department of Civil and Environmental Engineering, University of Illinois at Urbana-Champaign, 205 N. Mathews Ave., Urbana, IL 61801, U.S.A.

†E-mail: bfs@uiuc.edu

Newmark Endowed Chair of Civil Engineering.

${ }^{\S}$ Professor.
}

Contract/grant sponsor: National Science Foundation; contract/grant number: CMS 03-01140

Copyright (C) 2005 John Wiley \& Sons, Ltd.

Received 30 May 2005 Revised 6 September 2005 


\section{INTRODUCTION}

Structural health monitoring (SHM) and damage detection has become an important field in civil engineering, with the goal of continuous and periodic assessment of the safety and integrity of the civil infrastructure. Based on knowledge of the condition of the structure, certain preventative measures can be carried out to prolong the service life of the structure and prevent catastrophic failure. Detection of damage in the structure will ultimately reduce the life cycle costs.

Many algorithms have been developed for SHM. An excellent review of existing methods is found in References [1,2]. Therein, classification of SHM methods was based on the type of the measured data used, and/or the technique used to identify the damage. One category of these methods uses structural frequency changes. Vandiver [3] examined the change in resonant frequencies due to the damage in elements of the structure. More recently, Cha and Tuck-lee [4] examined the change in frequency response data; this information was then used to update the structural system parameters. Another category of these methods uses the change in mode shapes. West [5] was perhaps the first to implement systematic use of mode shape information for damage localization without the use of an a prior finite element model. After numerical and experimental study of a beam, Fox [6] suggested that graphical comparison of the mode shapes might be a good way to locate damage.

Another group of these methods takes advantage of the change in the flexibility matrix. Pandey and Biswas [7,8] presented a damage detection and localization method based on changes in the measured flexibility of the structure. Bernal [9] used the change of the flexibility matrix to compute a set of load vectors, having the property of inducing stress fields whose magnitudes are zero in the damaged areas. Other techniques include methods based on mode shape curvature changes [10,11], parameter estimation methods [12-14], neural network based methods [15,16], etc. In general, the existing methods require central acquisition and data processing, i.e. the measured data needs to be transferred to a central station, either through wired or wireless communication.

Damage in structures is an intrinsically local phenomenon. Responses from sensors close to the damaged site are expected to be more heavily influenced than those remote to the damage. Therefore, to effectively detect arbitrary damage in a complicated structure, a dense array of sensors distributed over the entire structure will be required. However, if the measured data is to be centrally acquired, significant limitations will exist for conducting SHM either using wired or wireless sensors. Use of traditional wired sensors is difficult due to the cost of deploying and maintaining a large wiring plant. Similarly, transferring all the measured information to a central station using wireless sensors is difficult because of power requirements and bandwidth limitations. In both cases, a tremendous amount of data is expected to be generated that would need to be sent to such a central station. Managing this large amount of data is challenging. Unnecessary information needs to be eliminated through data aggregation to efficiently utilize the network.

The recent development of the smart sensors has made health monitoring with a dense array of sensors feasible. A careful review of current smart sensing technologies has been provided by Spencer et al. [17,18]. The essential feature of a smart sensor is the on-board microprocessor, which grants these sensors the 'smart' characteristics. Code can be embedded in the sensor's microprocessor, which allows smart sensors to make decisions, perform computation, save data locally, etc. A portion of the computation can then be done at the sensor level for damage 
detection. Extraneous information can be discarded, reducing the information that needs to be transferred back to the central station. Note that all smart sensors to date are also wireless. Damage detection algorithms which can take advantage of the distributed computing environment offered by smart sensor technologies are needed, but currently limited.

This paper presents a distributed computing strategy (DCS) for SHM that is suitable for implementation on a densely distributed smart sensor network. In this proposed strategy, adjacent smart sensors are grouped together and only the locally measured information is used to evaluate the condition of local elements; the associated computation is done in a distributed manner. Damage detection results for these local elements can then be reported back to the central station. Continuous online monitoring of a structure can be done without relying on central data processing. In the sequel, a flexibility-based damage detection method that forms the basis for the SHM algorithm is first presented, followed by formulation of the flexibility matrix from both forced and ambient vibration. Extension of a flexibility-based method to handle the continuous online monitoring is then briefly reviewed. Damage detection using a limited number of senors localized in damaged regions is investigated, which leads to the proposed DCS approach for SHM. Finally, to demonstrate the efficacy of the DCS approach, verification by computer simulation using a 14-bay planar truss structure is provided.

\section{THE DAMAGE LOCATING VECTOR METHOD}

Damage detection methods based on the flexibility matrix have recently been shown to be quite promising. In particular, Bernal [9] proposed a flexibility-based damage localization method, the damage locating vector (DLV) method, which provides the foundation for the current work. The DLV method also has been experimentally verified by Gao et al. [19] and extended for continuous online monitoring employing ambient vibration by Gao and Spencer [20]. For completeness, the DLV method as employed herein is briefly reviewed in the section.

\subsection{Basic concept}

The DLV method is based on determination of a special set of load vectors, the so-called DLVs. The DLVs have the property that when they are applied to the structure as static forces at the sensor locations, no stress is produced in the damaged elements. This unique characteristic can be employed to localize structural damage.

Assuming nominally linear structural behaviour, the flexibility matrices at sensor locations are constructed from measured data before and after damage and denoted as $\mathbf{F}_{\mathbf{u}}$ and $\mathbf{F}_{\mathrm{d}}$, respectively. Then, all of the linear-independent load vectors $\mathbf{L}$ are collected which satisfy the following relationship:

$$
\mathbf{F}_{\mathrm{d}} \mathbf{L}=\mathbf{F}_{\mathrm{u}} \mathbf{L} \quad \text { or } \quad \mathbf{F}_{\Delta} \mathbf{L}=\left(\mathbf{F}_{\mathrm{d}}-\mathbf{F}_{\mathrm{u}}\right) \mathbf{L}=0
$$

This equation implies that the load vectors $\mathbf{L}$ produce the same displacements at the sensor locations before and after damage. From the definition, the DLVs are seen to also satisfy Equation (1); that is, because the DLVs produce no stress in the damaged structural elements, the damage of those elements does not affect the displacements at the sensor locations. Therefore, the DLVs are indeed the vectors in $\mathbf{L}$. 
To calculate $\mathbf{L}$, the singular value decomposition (SVD) is employed. The SVD of the matrix $\mathbf{F}_{\Delta}$ leads to

$$
\begin{gathered}
\mathbf{F}_{\Delta}=\mathbf{U S V} \mathbf{S}^{\mathrm{T}}=\left[\begin{array}{ll}
\mathbf{U}_{1} & \mathbf{U}_{0}
\end{array}\right]\left[\begin{array}{cc}
\mathbf{S}_{1} & 0 \\
0 & 0
\end{array}\right]\left[\begin{array}{ll}
\mathbf{V}_{1} & \mathbf{V}_{0}
\end{array}\right]^{\mathrm{T}} \\
{\left[\begin{array}{ll}
\mathbf{F}_{\Delta} \mathbf{V}_{1} & \mathbf{F}_{\Delta} \mathbf{V}_{0}
\end{array}\right]=\left[\begin{array}{ll}
\mathbf{U}_{1} \mathbf{S}_{1} & 0
\end{array}\right]}
\end{gathered}
$$

From Equation (3), one obtains

$$
\mathbf{F}_{\Delta} \mathbf{V}_{0}=0
$$

Equations (1) and (4) indicate that $\mathbf{L}=\mathbf{V}_{0}$, i.e. DLVs can be obtained from the SVD of the difference matrix $\mathbf{F}_{\Delta}$.

Each of the DLVs is then applied to an undamaged analytical model of the structure. The stress in each structural element is calculated and a normalized cumulative stress is obtained. The normalized cumulative stress for the $j$ th element is defined as

$$
\bar{\sigma}_{j}=\frac{\sigma_{j}}{\max _{k}\left(\sigma_{k}\right)} \quad \text { where } \sigma_{j}=\sum_{i=1}^{n} \operatorname{abs}\left(\frac{\sigma_{i j}}{\max _{k}\left(\sigma_{i k}\right)}\right)
$$

where $\sigma_{i j}$ is the stress in the $j$ th element induced by the $i$ th DLV and $\sigma_{j}$ the cumulative stress in the $j$ th element. If an element has zero normalized cumulative stress $\bar{\sigma}_{j}$, then this element is a possible candidate of damage. In practice, the normalized cumulative stresses induced by the DLVs in the damaged elements may not be exactly zero due to noise and uncertainties. Reasonable thresholds should be chosen to select the damaged elements [9].

\subsection{Construction of flexibility matrix}

As shown in the previous section, the flexibility matrices before and after damage need to be constructed to implement the DLV method. Depending on whether or not the input excitations are measured, different formulations are needed to construct flexibility matrix from the measured data. Both the cases are described here.

2.2.1. Formulation of flexibility matrix for the forced vibration case. When the input is measured and there is at least one co-located sensor and actuator pair, Bernal and Gunes [21] has shown how the experimental data can be used to obtain flexibility matrix for structures with general viscous damping. For a linear structure, the flexibility matrix can be expressed as

$$
\mathbf{F}=-\Psi \mathbf{D}_{\mathrm{g}} \Psi^{\mathrm{T}}
$$

where $\Psi$ is the arbitrarily normalized complex mode shapes, $\mathbf{D}_{\mathrm{g}}$ the $\operatorname{diag}\left(\left[d_{1}, d_{2}, \ldots, d_{j}, \ldots\right]\right)$, and $d_{j}$ the modal normalization constant.

To obtain the quantities in Equation (6), a state space representation of the structure can be obtained from the measured data using various system identification algorithms, such as the eigensystem realization algorithm (ERA) [22]. By converting the discrete time state space representation identified from the measured data to continuous time, one obtains

$$
\begin{aligned}
\dot{\mathbf{z}} & =\mathbf{A z}+\mathbf{B u} \\
\mathbf{y} & =\mathbf{C} \mathbf{z}+\mathbf{D u}
\end{aligned}
$$


where $\mathbf{z}=$ state vector; $\mathbf{u}=$ input excitation vector; $\mathbf{y}=$ vector of measured outputs; $\mathbf{A}=$ state matrix; $\mathbf{B}=$ input influence matrix; $\mathbf{C}=$ output influence matrix; and $\mathbf{D}=$ direct transmission matrix. Transforming Equation (7) to the frequency domain and noting that the flexibility matrix relates the inputs to the outputs at $\omega=0$, the flexibility matrix can be obtained as

$$
\mathbf{F}_{\mathrm{f}}=-\mathbf{C} \varphi \lambda^{-(p+1)} \varphi^{-1} \mathbf{B}=-\Psi_{\mathrm{m}} \lambda^{-(p+1)} \varphi^{-1} \mathbf{B}
$$

where $p=0,1$, and 2 when measured outputs are displacement, velocity, and acceleration, respectively; $\lambda$ and $\varphi=$ eigenvalues and eigenvectors of matrix $\mathbf{A}$; and $\Psi_{\mathrm{m}}=\mathbf{C} \varphi$ and $\Psi_{\mathrm{m}}=$ complex mode shapes at sensor locations.

The $i$ th column of $\mathbf{F}_{\mathrm{f}}$ represents the displacements of the structure at the sensor locations due to a unit force being applied at the $i$ th excitation location, $u_{i}$. If we denote the flexibility matrix at the sensor locations as $\mathbf{F}_{\mathrm{m}}$, the $j$ th column of $\mathbf{F}_{\mathrm{m}}$ represents the displacements of the structure at the sensor locations due to a unit force being applied at the jth sensor location. If any of the inputs are co-located with sensors, then the corresponding columns in the matrices $\mathbf{F}_{\mathrm{f}}$ and $\mathbf{F}_{\mathrm{m}}$ will be equal. Defining two Boolean matrices $\mathbf{q}_{\mathrm{f}}$ and $\mathbf{q}_{\mathrm{m}}$ which pick out these columns from $\mathbf{F}_{\mathrm{f}}$ and $\mathbf{F}_{\mathrm{m}}$, respectively, we have

$$
-\Psi_{\mathrm{m}} \mathbf{D}_{\mathrm{g}} \Psi_{\mathrm{m}}^{\mathrm{T}} \mathbf{q}_{\mathrm{m}}=-\Psi_{\mathrm{m}} \lambda^{-(p+1)} \varphi^{-1} \mathbf{B} \mathbf{q}_{\mathrm{f}}
$$

The modal normalization constant $d_{j}$ can then be solved from Equation (9) as

$$
d_{j}=\lambda_{j}^{-(p+1)} \bar{\varphi}_{j}^{\mathrm{T}} \mathbf{B}\left[\operatorname{diag}\left(\Psi_{\mathrm{m}, j}^{\mathrm{T}} \mathbf{q}_{\mathrm{m}}\right)\right]^{-1}
$$

where $\bar{\varphi}_{j}^{\mathrm{T}}=j$ th row of matrix $\varphi^{-1}$; and $\Psi_{\mathrm{m}, j}^{\mathrm{T}}=j$ th row of matrix $\Psi_{\mathrm{m}}^{\mathrm{T}}$. When there is more than one co-located sensor and actuator pair, multiple estimations of $d_{j}$ will be obtained. Bernal and Gunes [21] suggested that the $d_{j}$ corresponding to the component in vector $\Psi_{\mathrm{m}, j}^{\mathrm{T}} \mathbf{q}_{\mathrm{m}}$ with the largest magnitude might be used.

2.2.2. Formulation of flexibility matrix for the ambient vibration case. For ambient vibration case, a mass perturbation method for construction of flexibility matrix suggested by Bernal [23] will be presented in this section. Recall that for a linear structure with classical damping, the flexibility matrix takes the form

$$
\mathbf{F}=(\psi \alpha) \Lambda^{-1}(\psi \alpha)^{\mathrm{T}}
$$

where $\psi=$ undamped arbitrarily normalized mode shapes; $\Lambda$ and $\psi=$ solutions of the eigenproblem $\mathbf{K} \psi=\mathbf{M} \psi \Lambda$; $\alpha$ the $\operatorname{diag}\left(\left[\alpha_{1}, \alpha_{2}, \ldots, \alpha_{j}, \ldots\right]\right) ;$ and $\alpha_{j}=$ modal, normalization constant.

To obtain $\alpha_{j}$ for the ambient vibration case, the mass matrix of the modified structure can be expressed as

$$
\mathbf{M}_{1}=\mathbf{M}_{0}+\Delta \mathbf{M}
$$

in which $\mathbf{M}_{0}=$ mass matrix of the original structure, $\mathbf{M}_{1}=$ mass matrix of the modified structure; $\Delta \mathbf{M}=$ matrix describing the mass perturbation. The eigenvalue problem for the modified structure is

$$
\mathbf{K} \psi_{1, j}=\lambda_{1, j}\left(\mathbf{M}_{0}+\Delta \mathbf{M}\right) \psi_{1, j}
$$

where $\lambda_{1, j}=j$ th eigenvalue of the modified structure; and $\psi_{1, j}=j$ th eigenvector of the modified structure. The corresponding eigenvalues and eigenvectors for the original structure 
are $\lambda_{0, j}$ and $\psi_{0, j}$, respectively. The mode shape $\psi_{1, j}$ can be written as

$$
\psi_{1, j}=\psi_{0} \mathbf{q}_{j}+N\left(\psi_{0}\right) \mathbf{g}_{j}
$$

where $\psi_{0}=\left[\psi_{0,1}, \psi_{0,2}, \ldots, \psi_{0, j}, \ldots\right], N\left(\psi_{0}\right)=$ column null space of $\psi_{0}$; and $\mathbf{q}_{j}$ and $\mathbf{g}_{j}=$ coefficient vectors. Substituting Equation (14) into Equation (13) and multiplying both sides by $\psi_{0}^{\mathrm{T}}$ yields

$$
\alpha^{2}\left(\mathbf{R}_{j}+\varepsilon_{j}\right)=\Lambda_{0} \mathbf{q}_{j}-\lambda_{1, j} \mathbf{q}_{j}
$$

where

$$
\begin{aligned}
& \mathbf{R}_{j}=\lambda_{1, j} \psi_{0}^{\mathrm{T}} \Delta \mathbf{M} \psi_{1, j} \\
& \varepsilon_{j}=\lambda_{1, j} \psi_{0}^{\mathrm{T}} \mathbf{M}_{0} N\left(\psi_{0}\right) \mathbf{g}_{j}-\psi_{0}^{\mathrm{T}} \mathbf{K} N\left(\psi_{0}\right) \mathbf{g}_{j}
\end{aligned}
$$

with $\Lambda_{0}=\operatorname{diag}\left[\lambda_{0,1}, \lambda_{0,2}, \ldots, \lambda_{0, j}, \ldots\right]$ and $\mathbf{q}_{j}=\left(\psi_{0}^{\mathrm{T}} \psi_{0}\right)^{-1} \psi_{0}^{\mathrm{T}} \psi_{1, j}$. By neglecting the error term $\varepsilon_{j}$, Equation (15) can be rewritten as

$$
\alpha^{2} \lambda_{1, j} \psi_{0}^{\mathrm{T}} \Delta \mathbf{M} \psi_{1, j}=\Lambda_{0} \mathbf{q}_{j}-\lambda_{1, j} \mathbf{q}_{j}
$$

which can be de-coupled to solve for the unknown $\alpha_{i}$ as

$$
\alpha_{i}^{2}=\frac{\lambda_{0, i}-\lambda_{1, j}}{\lambda_{1, j}} \frac{q_{i j}}{\psi_{0, j}^{\mathrm{T}} \Delta \mathbf{M} \psi_{1, j}}
$$

The modal parameters for both the original and modified structure can be obtained employing the natural excitation technique (NExT) [24] in conjunction with the ERA method. Equation (18) indicates that there is one set of $\left[\alpha_{1}, \alpha_{2}, \ldots, \alpha_{i}, \ldots\right]$ for each $\psi_{1, j}$ i.e. there are various estimations for different $\psi_{1, j}$. Bernal [23] pointed out that the most accurate estimation for the normalization index $\alpha_{i}$ is when $i=j$ in Equation (18).

\subsection{Extending the DLV method for continuous online SHM}

The DLV method is not easily employed for continuous online damage diagnosis. Equations (10) and (18) shows that the modal normalization constants $d_{j}$ or $\alpha_{j}$ need to be obtained to construct the flexibility matrix. As suggested in the derivation of Equations (10) and (18), a certain degree of interruption of structural operation will be needed to obtain these normalization constants from measured data. The method based on forced vibration requires employing an external exciter, e.g. an impactor or a rotating imbalance vibrator, to shake the structure. The method based on ambient vibration requires adding mass to the structure to conduct dynamic testing. These methods can be used to compute the undamaged normalization constants. However, computing the damaged normalization constants by one of these methods each time the health of the structure is assessed is intractable. An approach which can take the advantage of the DLV method and is suitable for continuous online monitoring is required.

Gao and Spencer [20] proposed an algorithm which extends the DLV method for continuous online monitoring. The essence of the approach is to construct an approximate flexibility matrix for the damaged structure utilizing the undamaged normalization constants with the mode shapes before and after damage being normalized to have a unit magnitude.

The first step of the proposed algorithm is to compute the undamaged normalization constants and then the undamaged flexibility matrix from the measured data. This step is termed algorithm initialization. Once the initialization is completed, the second step of the proposed 

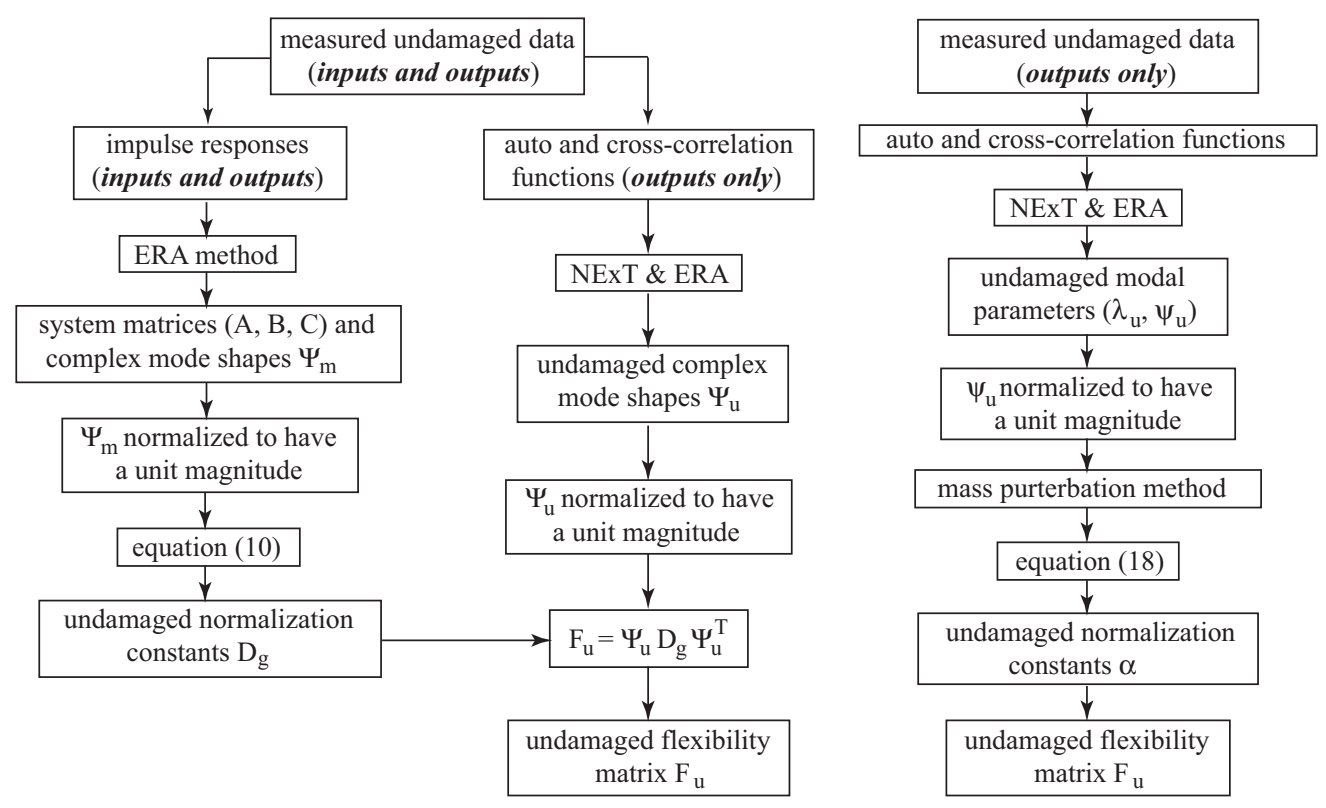

Figure 1. Algorithm initialization.

approach is to construct an approximate flexibility matrix for the potentially damaged structure employing ambient vibration and then to apply the DLV method to detect damage in the structure. Herein, this step is referred as algorithm operation. The flow charts for the algorithm initialization and operation are shown in Figures 1 and 2. This extended DLV method is incorporated into the proposed DCS approach, which is presented in Section 4.

For more detailed information regarding the DLV method, the interested reader is directed to References [9,19,20].

\section{DAMAGE DETECTION USING LIMITED SENSOR INFORMATION}

In this section, damage detection using a limited number of sensors distributed throughout the structure or localized in damaged regions are investigated. These results lead directly to the DCS approach proposed in Section 4.

\subsection{Sensors distributed throughout the structure}

To investigate damage detection using a limited number of sensors distributed throughout the structure, a numerical example employing a 14-bay planar truss (Figure 3) is considered. A Matlab finite element model consisting of 53 bars and 28 nodes was developed. Each structural node number has a circle around it.

In this example, first four mode shapes in the $y$-direction evaluated at all the lower chord nodes except the supports are used. Two $13 \times 13$ flexibility matrices before and after damage can 


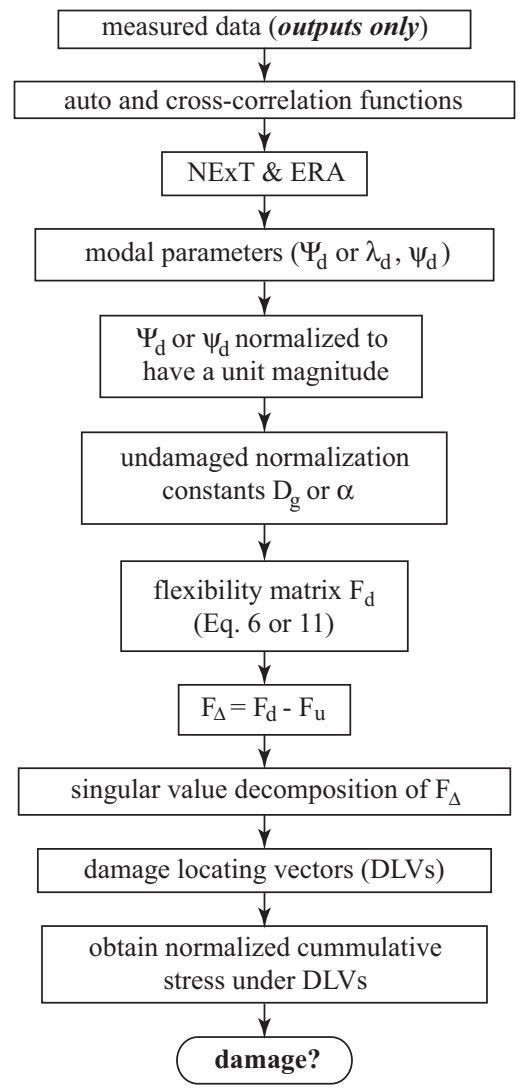

Figure 2. Algorithm operation.

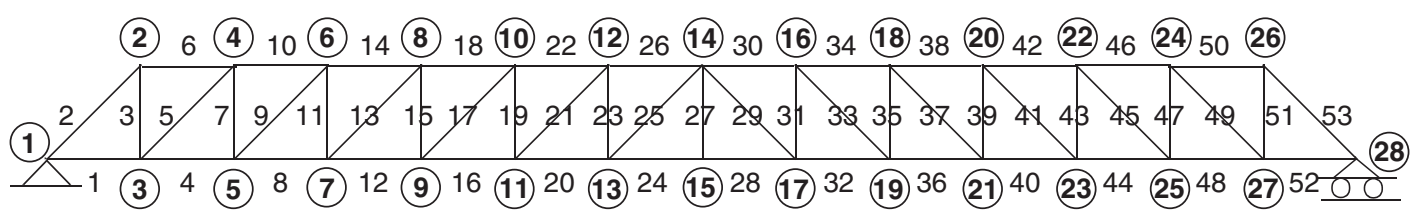

Figure 3. 14-bay planar truss.

be obtained from the exact numerical model. The DLV method is then applied to locate the damage.

Figure 4 shows the results when element 5 is damaged. As can be seen, elements 5 and 7 have considerably smaller normalized cumulative stresses as compared with other elements; therefore they are identified as possibly damaged elements. Under the current load configuration where only the vertical loads are applied at the lower chord nodes, if either one of these two elements has a small axial stress, so does the other. This result can be easily understood from the force equilibrium in $y$-direction of node 4 . 


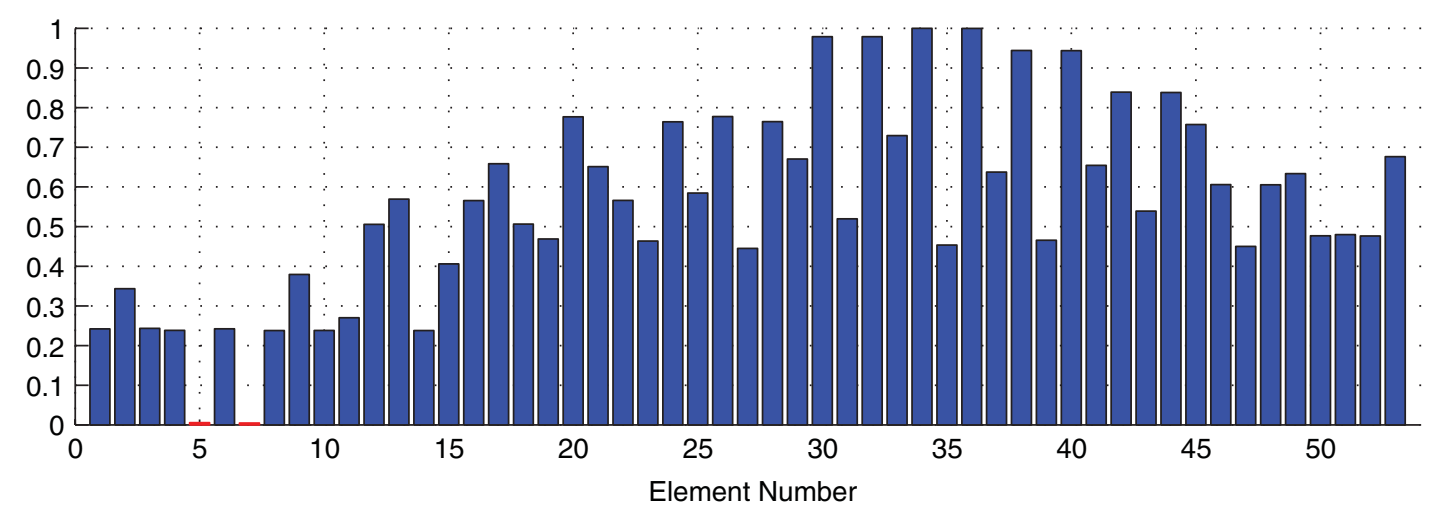

Figure 4. Normalized cumulative stress when element 5 has a $20 \%$ stiffness reduction.

As shown in Figure 4, good numerical results have been obtained using a limited number of sensors distributed throughout the structure; however, questions arise about whether the sensor information away from the damaged region are indeed needed. Can we only use the information from the sensors in the vicinity of the damaged region to locate the damage? This question is addressed in the next section.

\subsection{Sensors localized in damaged region}

The potential of locating damage using only a limited number of sensors localized in the damaged region can be seen by further consideration of the planar truss shown in Figure 3. For this truss, sensors on every three consecutive lower chord nodes and the corresponding upper chord nodes are grouped together to monitor the elements connected to these nodes. These groups are formed from left to right in the truss structure. To allow some computational redundancy, every two groups have some overlap. For example, group 1 includes nodes [2 345 6 7], and group 2 includes structural nodes $\left[\begin{array}{llllll}4 & 5 & 6 & 7 & 8 & 9\end{array}\right]$, etc. Therefore, for each group of localized sensors there are a total of 12 sensors which are installed in the $x$-and $y$-direction at six structural nodes. And there are a total of 11 different localized sensor groups.

In this initial example, system identification is not employed; rather, the exact modal parameters (i.e. mode shapes and frequencies) are used to constructed truncated flexibility matrix which is then employed in the DLV method. The flexibility matrix at the sensor locations is constructed using the first four modes. The normalized cumulative stress of all the structural members is computed for each localized sensor group and shown in Figure 5. Results from group 1 to 11 are shown in the order from top to the bottom. In each plot, a circle is used to mark elements in which both nodes are members of the group, and a cross signifies the elements in which only one of its node is a member of a group. Only the condition of elements in which both nodes are in a given group are evaluated here for damage by the localized sensor group.

Results in group 1 shows that element 5 has a very small normalized cumulative stress compared with other elements in the group, which indicates that element 5 is a possibly damaged element. The results from all other groups indicate that there is no other damaged element in this truss. It has been demonstrated that the damaged element, in this case element 5 , 


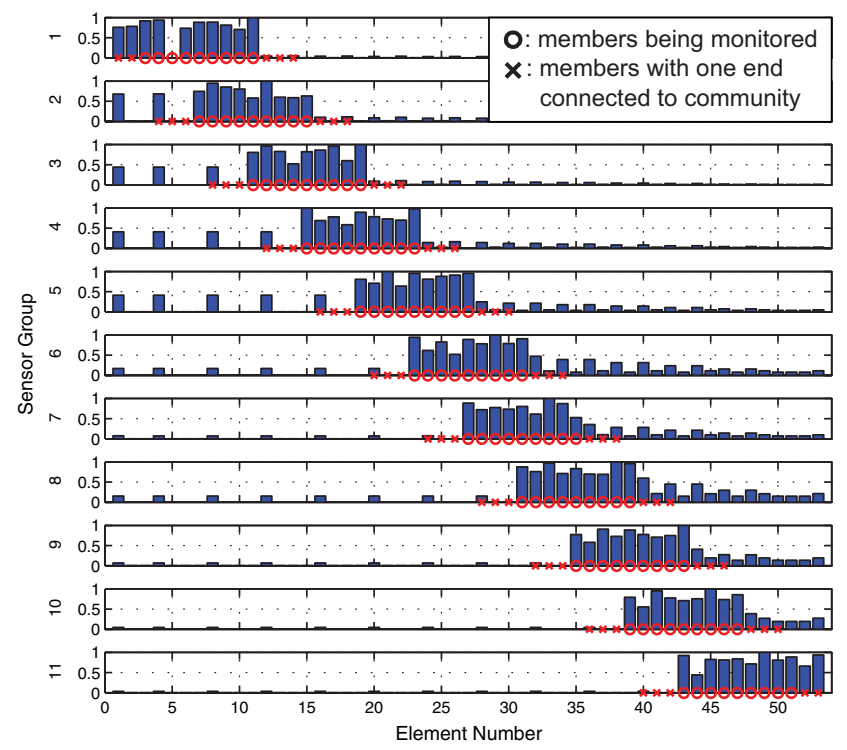

Figure 5. Normalized cumulative stress when element 5 has $20 \%$ stiffness reduction.

can be determined based on information of a small group of sensors localized in the damaged region. This result implies that the information away from the damage location might not be necessary for damage detection. This concept can be employed for damage localization for structures with a dense array of sensors.

\section{DISTRIBUTED COMPUTING STRATEGY (DCS)}

\subsection{Hierarchical organization}

The conceptual hierarchical organization of the DCS approach is shown in Figure 6. In contrast to traditional SHM algorithms which require all the measured information to be transferred to a central station, the measured information is aggregated locally by a selected sensor within the sensor group, termed the manager sensor, and only limited information is sent back to the central station to provide the condition of the structure. Small numbers of smart sensors are grouped to form different communities. For clarity, this figure shows each sensor as being included in only one community; however, in the proposed approach, each sensor can participate in multiple communities. For each community, the manager sensor collects measured responses and implements the damage detection algorithm for this community. Adjacent manager sensors need to interact with each other to exchange information. Referring again to Figure 6, manager sensors in communities 1, 2, and 3 interact with each other while community 4 only interacts with community 3 .

After the measured information is aggregated locally, the manager sensor determines what information needs to be sent back to the central station. In the proposed approach, each of the 


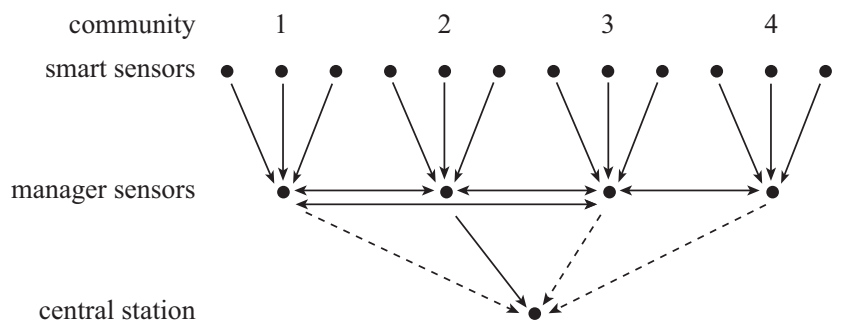

Figure 6. Sketch of hierarchical organization.

communities in which damage has not occurred only transmits an 'ok' signal to the central station, which is reflected by the dotted line connection in Figure 6. The communities in which damage has occurred need to send information about the damaged elements, which is reflected by the solid line connection in Figure 6. In this way, only limited information needs to be transferred between sensors throughout the entire structure. This approach will significantly reduce the communication traffic and the associated power demands in the sensor network.

\subsection{Strategy implementation}

The planar truss structure shown in Figure 3 is employed to illustrate the details of the implementation of the proposed SHM strategy.

4.2.1. Community development. First, the sensor communities are formed. A single community includes a set of adjacent structural nodes, sensors on these nodes, and members. These structural members have both ends connected to the structural nodes in the same community. Figure 7 shows an example of how communities can be formed. Different communities are developed from left to right in the truss structure. To facilitate efficient communication among the smart sensors, the structural nodes within the same community should be close to each other. As an example, community 6 in Figure 7 includes nodes [12 13141516 17] and elements [23 24252627282930 31].

Only the elements which have both ends connected to community nodes are monitored by the manager sensor for this community. There is an exception for those communities close to the supports. If a structural member has one end connected to the support and the other end connected to a community node, then this structural member can also monitored by this community. For example, in Figure 7, elements 1 and 2 only have one end connected to the community nodes, but they both have the other end connected to a support; therefore these two elements will be investigated by the smart sensors in community 1 .

To allow some computational redundancy, adjacent communities are recommended to have some overlaps so that each structural member is monitored by more than a single community.

4.2.2. Computing undamaged normalization constants for communities. Once the community is formed, the modal normalization constants for the undamaged structure have to be computed employing either forced or ambient vibration. For this purpose, one sensor from each community, termed the reference sensor, will be required to send the recording data to the 


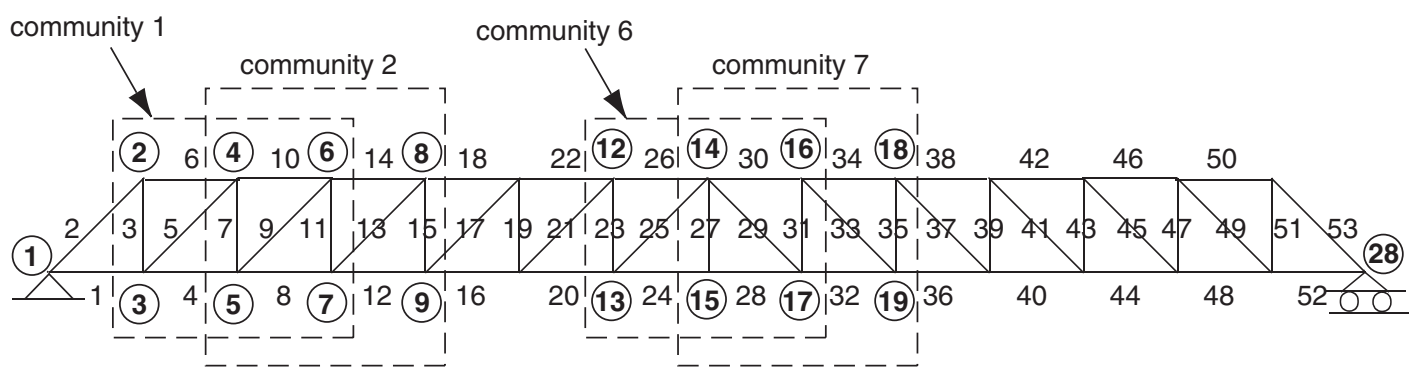

Figure 7. Forming community for damage detection.

central station. These reference sensors are selected such that their mode shape magnitudes will not be zero for the modes of interest. The modal parameters associated with these reference sensors, denoted as $\hat{\Psi}_{j}$ or $\hat{\lambda}_{j}$ and $\hat{\psi}_{j}$, respectively, are identified using the ERA method or the NExT in conjunction with the ERA method. The undamaged normalization constants can then be computed based on the methods presented in Section 2.2, and denoted as $\hat{d}_{j}$ or $\hat{\alpha}_{j}$.

However, the normalization constants $\hat{d}_{j}$ or $\hat{\alpha}_{j}$ cannot be employed directly by each community for damage detection, because the scalar for the $j$ th mode shape $\hat{\Psi}_{j}$ or $\hat{\psi}_{j}$ and the $j$ th mode shape $\Psi_{j}^{i}$ or $\psi_{j}^{i}$ in $i$ th community can be different. The $j$ th undamaged normalization constant for the $i$ th community $d_{j}^{i}$ or $\alpha_{j}^{i}$ can be obtained as

$$
d_{j}^{i}=\hat{d}_{j}\left(\frac{\hat{\Psi}_{j}(i)}{\Psi_{j}^{i}(k)}\right)^{2} \quad \text { and } \quad \alpha_{j}^{i}=\hat{\alpha}_{j} \cdot \frac{\hat{\psi}_{j}(i)}{\psi_{j}^{i}(k)}
$$

where $j=j$ th mode; $i=i$ th community; and $k=$ reference sensor location in the $i$ th community. The undamaged normalization constants for the $i$ th community are $\mathbf{D}_{g}^{i}=$ $\left[d_{1}^{i}, d_{2}^{i}, \ldots, d_{j}^{i}, \ldots\right]$ or $\alpha^{i}=\left[\alpha_{1}^{i}, \alpha_{2}^{i}, \ldots, \alpha_{j}^{i}, \ldots\right]$.

4.2.3. Data aggregation. To minimize the communication traffic in the sensor network, measured data need to be transferred to the manager sensor for data processing.

Clocks of smart sensors in the same community are first synchronized with each other. The measurements are then collected, which will need to be transferred to the manager sensor for computation. To facilitate communication, the manager sensor should be centrally located to the other sensors in the community. For example, in Figure 8, the sensors at structural node 15 is selected as the manager sensor for community 6 . As can be seen from the figure, some sensors may need to transfer information to more than one manager sensors. This situation occurs when the smart sensors participate in different communities.

After the data have been transferred to the manager sensor, computation can be conducted using the on-board microprocessor to locate the damage within the community. The extended DLV method presented in Section 2.3 is incorporated in this SHM strategy to localize the damage in each community. The flow chart for damage aggregation in a community is shown in Figure 9. Note that the undamaged flexibility matrix $F_{u}$ in Figure 9 is constructed using Equation (6) or (11) once the undamaged modal normalization constants $d_{j}^{i}$ or $\alpha_{j}^{i}$ are computed for community $i$. 

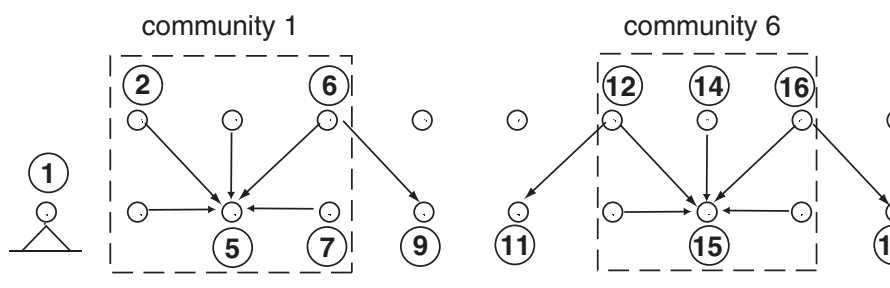

Figure 8. Data collection.

4.2.4. Decision making. Actions need to be taken after the data aggregation is done for a community. If there is no damage detected in a community, the manager sensor does not initiate interaction with other manager sensors; rather, it simply sends in 'ok' signal back to the central station. If there is damage identified in a community, the manager sensor needs to send queries to its counterpart in adjacent communities. There are three possibilities after sending the queries:

- The damage candidate in community $i$ does not participate in adjacent communities. The manager sensor in community $i$ sends the damage information back to the central station.

- The damage candidate in community $i$ participates in other communities and identified as the potentially damaged location in all of these communities. This damage candidate is then confirmed and reported to the central station by these communities.

- The damage candidate identified by community $i$ participates in other communities, but not all of the communities identify it as the potentially damaged element. These communities then need to retake data, and re-conduct data aggregation and decision making.

A flow chart for the decision making is shown in Figure 9.

To better illustrate how the proposed decision making algorithm works, a simple example is shown in Figure 10. The manager sensors in each community first conduct damage detection locally. Those communities having no damage send 'ok' signal back to the central station. Communities 3, 4, and 8 interact with the surrounding communities because damage is detected in these communities. As a result, communities 3 and 4 confirm that element 16 is the damage location and send this damage information back to the central station. Communities 7 and 8 are required to retake data and re-conduct damage detection as inconsistent information has been obtained regarding element 33 . Results regarding element 33 need to be reported to the central station after re-conducting damage detection.

\section{NUMERICAL VALIDATION}

The proposed DCS approach is verified using the planar truss structure shown in Figure 3. This planar truss consists of 53 steel circular bars, which have a cross section of $1.122 \times 10^{-4} \mathrm{~m}^{2}$ and a moment of inertial of $2.111 \times 10^{-9} \mathrm{~m}^{4}$. The elastic modulus of the material is $2 \times 10^{11} \mathrm{~N} / \mathrm{m}^{2}$ and the mass density is $7.83 \times 10^{3} \mathrm{~kg} / \mathrm{m}^{3}$. The total length of this truss is $5.6 \mathrm{~m}$ with $0.4 \mathrm{~m}$ in each bay, and the truss height is $0.4 \mathrm{~m}$. Members and structural nodes are numbered same as Figure 3. 


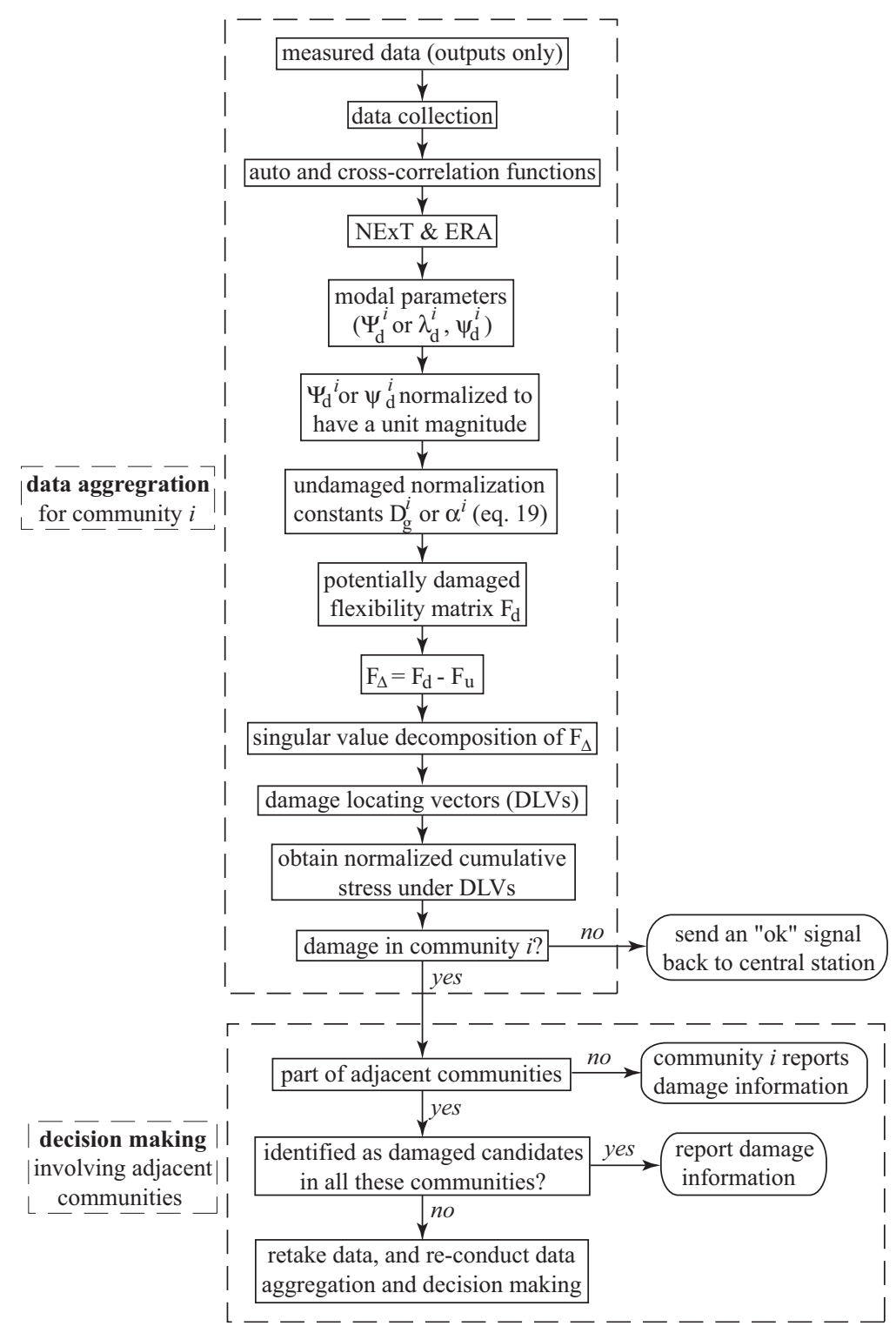

Figure 9. Data aggregation and decision making.

Accelerometers are installed in the $x$ - and $y$-direction at all the nodes except the supports. Communities are developed as described in Section 4.2. A band-limited white noise with an RMS amplitude around 5\% of the measured signal is added to simulate measurement noises. Only the first three modes identified from the measured data are utilized for damage detection.

This truss structure is excited in the $y$-direction using two independent band-limited white noises. To better assess efficacy of the proposed approach in practice, both excitation location 


\begin{tabular}{|c|c|c|c|c|c|c|c|c|}
\hline & \multirow{2}{*}{ damage } & \multicolumn{2}{|c|}{ action } & \multicolumn{2}{|c|}{ action } & \multicolumn{2}{|c|}{ action } & \\
\hline & & send & receive & send & receive & send & receive & \\
\hline G1 & No & S "ok" B & - & SLE & EPP & & & $\longrightarrow$ \\
\hline G2 & No & S "ok" B & $\mathrm{R}$ ?16 G3 & S "0" G3 & - & SLEE & EP & $\longrightarrow$ \\
\hline \multirow{2}{*}{ G3 } & \multirow{2}{*}{ Yes } & $\mathrm{S} ? 16 \mathrm{G} 2$ & - & - & R "0" G2 & \multirow{2}{*}{ S "D16" B } & \multirow{2}{*}{ - } & \\
\hline & & $\mathrm{S}$ ?16 G4 & $\mathrm{R}$ ?16 G4 & S "1" G4 & R "1" G4 & & & \\
\hline \multirow{2}{*}{ G4 } & \multirow{2}{*}{ Yes } & S ?16 G3 & R ?16 G3 & S "1" G3 & R "1" G3 & \multirow{2}{*}{ S "D16" B } & \multirow{2}{*}{ - } & \\
\hline & & S ? 16 G5 & - & - & R "0" G5 & & & \\
\hline G5 & No & S "ok" B & $\mathrm{R}$ ? $16 \mathrm{G} 4$ & S "0" G4 & - & SLEE & & $\rightarrow$ \\
\hline G6 & No & S "ok" B & - & SLE & EPP & & & $\rightarrow$ \\
\hline G7 & No & S "ok" B & R ?33 G8 & S "-1" G8 & - & - & R "C" G8 & P "C" \\
\hline \multirow{2}{*}{ G8 } & \multirow{2}{*}{ Yes } & S ?33 G7 & - & - & R "-1" G7 & S "C" G7 & \multirow{2}{*}{ - } & \multirow{2}{*}{ P "C" } \\
\hline & & S ?33 G9 & - & - & R "0" G9 & S "I33 7-8" B & & \\
\hline G9 & No & S "ok" B & $\mathrm{R}$ ?33 G8 & S "0" G8 & - & SLEE & EP & $\longrightarrow$ \\
\hline G10 & No & S "ok" B & - & SLE & EPP & & & $\rightarrow$ \\
\hline G11 & No & S "ok" B & - & SLE & EPP & & & $\longrightarrow$ \\
\hline
\end{tabular}

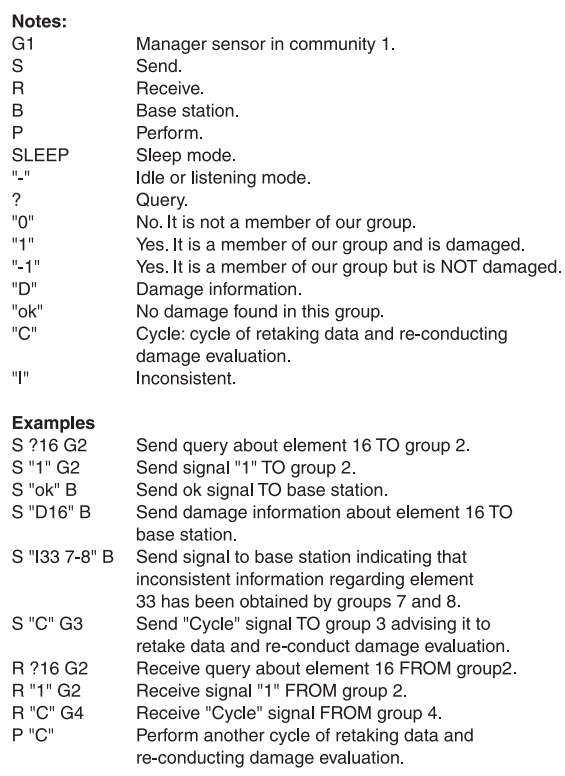

Figure 10. Decision making example (element 16 is consistently identified as having damage in communities 3 and 4; and inconsistent information has been obtained by communities 7 and 8 regarding element 33).

and magnitude have been changed before and after damage. For algorithm initialization, these two excitations are at nodes 7 and 11 with a magnitude of power spectral density (PSD) of 0.12 and 0.18 , respectively. For algorithm operation, i.e. damage detection, these two excitations are at nodes 9 and 23 with a height of PSD of 0.18 and 0.09 . These two excitations are not measured except for the case of algorithm initialization employing forced vibration.

Two damage cases are considered:

Case 1: single damage scenario-20\% stiffness reduction at element 13 .

Case 2: multiple damage scenario-20\% stiffness reduction in elements 17 and 36 .

\subsection{Constructing undamaged flexibility matrix in communities}

The undamaged normalization constants $\hat{d}_{j}$ or $\hat{\alpha}_{j}$ in Equation (19) are first obtained using the methods outlined in Section 2.2 from forced or ambient vibration. Then the SHM system can start measuring data to construct the undamaged flexibility matrix employing ambient vibration.

First, the acceleration measurements are collected and the auto- and cross-spectral density functions are computed by selecting a reference output, which are used to compute the corresponding auto- and cross-correlation functions. The sample correlation function, between the acceleration in the $y$-direction of node 5 and node 7 (reference output in community 1), is shown in Figure 11. These correlation functions can then be used by the ERA method to extract 


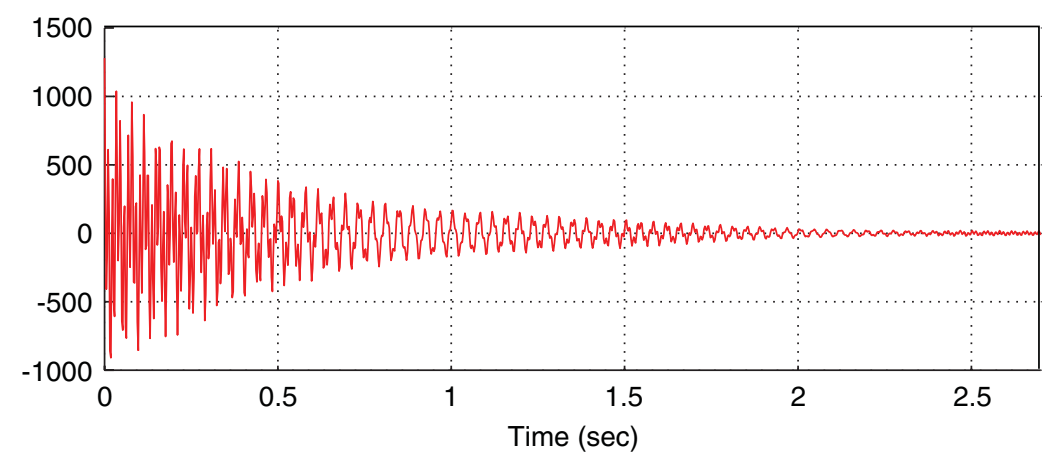

Figure 11. Correlation function.

Exact: $25.9587 \mathrm{~Hz}$ Estimated: $25.9657 \mathrm{~Hz}$

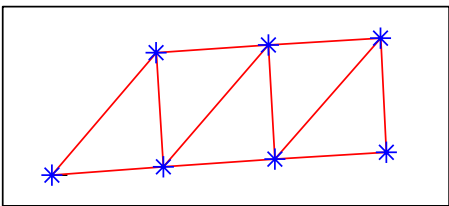

Exact: $88.0730 \mathrm{~Hz}$ Estimated: $88.0758 \mathrm{~Hz}$

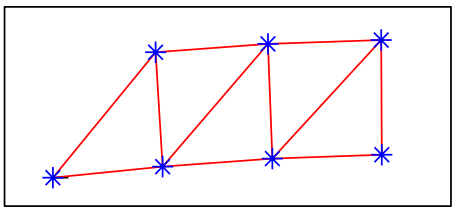

Exact: $126.3466 \mathrm{~Hz}$ Estimated: $126.4384 \mathrm{~Hz}$

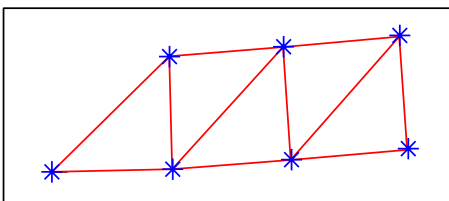

Figure 12. Modal parameters.

the modal parameters and sample results are shown in Figure 12. In this figure, the star represents the identified mode shape, and the solid line represents exact mode shape from the undamaged analytical model. As can be seen, system identification results using only outputs have shown good agreement with the analytical model.

Once the undamaged modal parameters in each community are identified, the associated normalization constants can be obtained using Equation (19), and the undamaged flexibility matrix can then be constructed utilizing Equation (6) or (11). Comparison of the identified flexibility matrices with the exact one at the measured DOFs in the $x$-and $y$-direction of nodes 3 
Table I. Comparison of the undamaged flexibility matrices using the first three modes $\left(\times 10^{-6} \mathrm{~m} / \mathrm{N}\right)$.

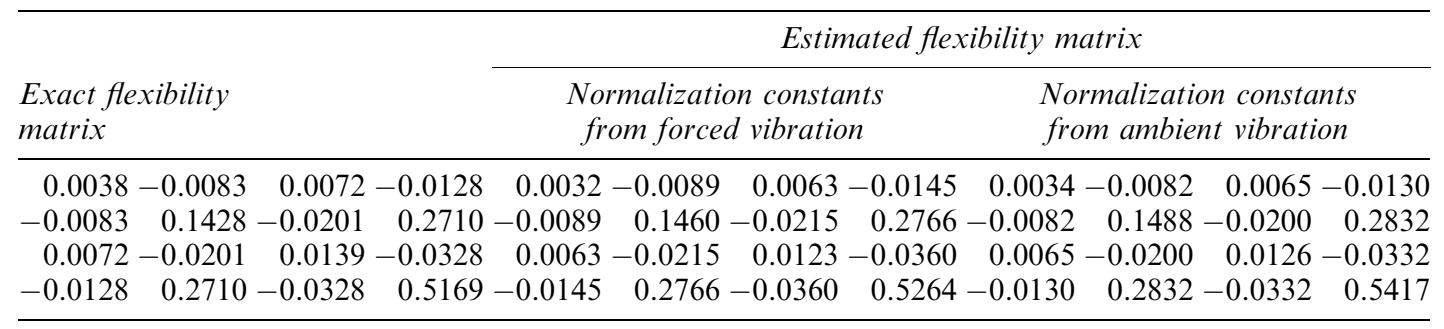

and 5 is shown in Table I. As can be seen, good results have been obtained using the modal normalization constants identified from both forced and ambient vibration.

\subsection{Damage detection results}

As stated in Section 2.1, the normalized cumulative stresses induced by the DLVs in the damaged elements may not be exactly zero due to noise and uncertainties, and reasonable thresholds should be chosen to select the damaged elements. For this truss structure, a value of 0.3 has been found working well for a wide range of damage scenarios while using a total of seven DLVs associated with the smallest singular values. This cut-off value is therefore utilized here to select the damaged elements.

5.2.1. Single damage scenario. By constructing an approximate flexibility matrix for each community employing the locally measured information, the DLV method can be applied for continuous monitoring of the structure. The results of the normalized cumulative stress for each community when element 13 has a $20 \%$ stiffness reduction is displayed in Figure 13. In both cases, results from communities 2 and 3 show that the normalized cumulative stress for element 13 is considerably smaller than other elements being monitored and smaller than the threshold. Therefore, this element is confirmed as a damage location in these communities. Results from other communities show no elements having a small normalized cumulative stress. The manager sensors in communities 2 and 3 send the damage information to the central station, while other communities only send an 'ok' signal back to the central station.

5.2.2. Multiple damage scenario. Figure 14 shows the results when elements 17 and 36 have a $20 \%$ stiffness reduction. Again, communities 3 and 4 determine element 17 as a possibly damaged element; communities 8 and 9 identify element 36 as a damage candidate.

For the case of initialization based on forced vibration, community 4 also reports element 23 as a damage candidate as its normalized cumulative stress is smaller than the threshold. However, communities 5 and 6 determine it as undamaged. Inconsistent information has been obtained from communities 4,5 , and 6 regarding element 23 . Therefore, communities 4,5 , and 6 need to retake data and re-conduct damage detection. The results are shown in Figure 15 which identifies element 23 as an undamaged location.

In both cases, communities 3, 4, 8, and 9 report the damage information back to the central station; while other communities having no damage only send back an 'ok' signal. 


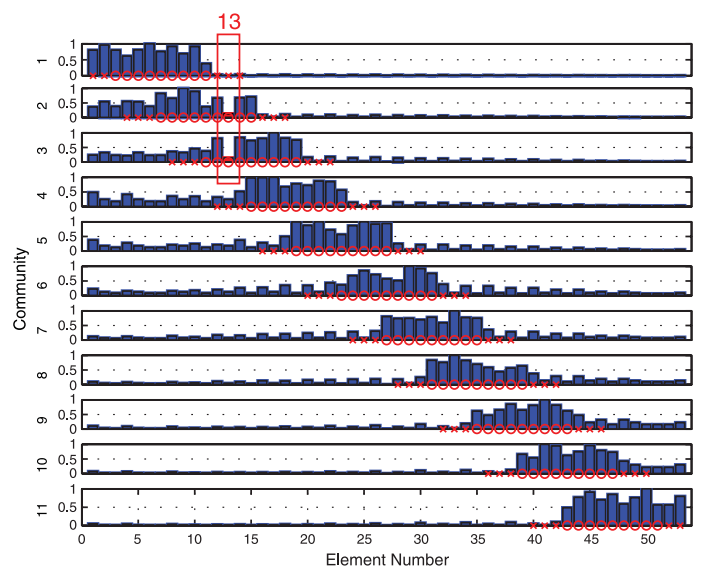

Initialization based on forced vibration

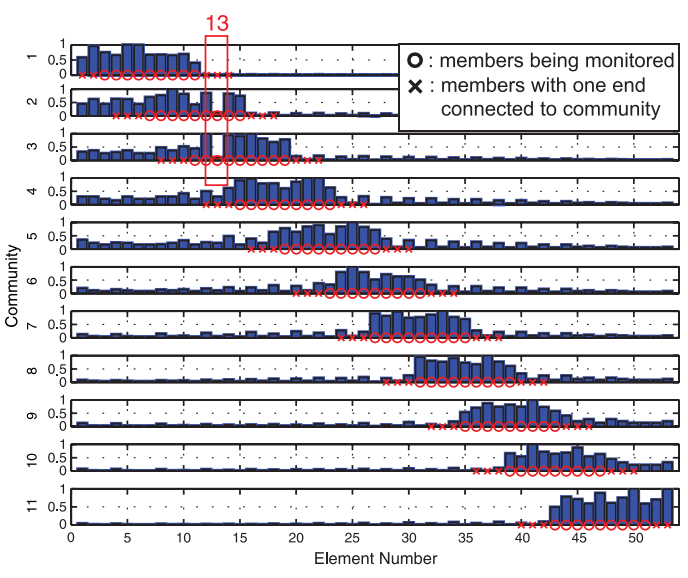

Initialization based on ambient vibration

Figure 13. Normalized cumulative stress when element 13 is damaged.
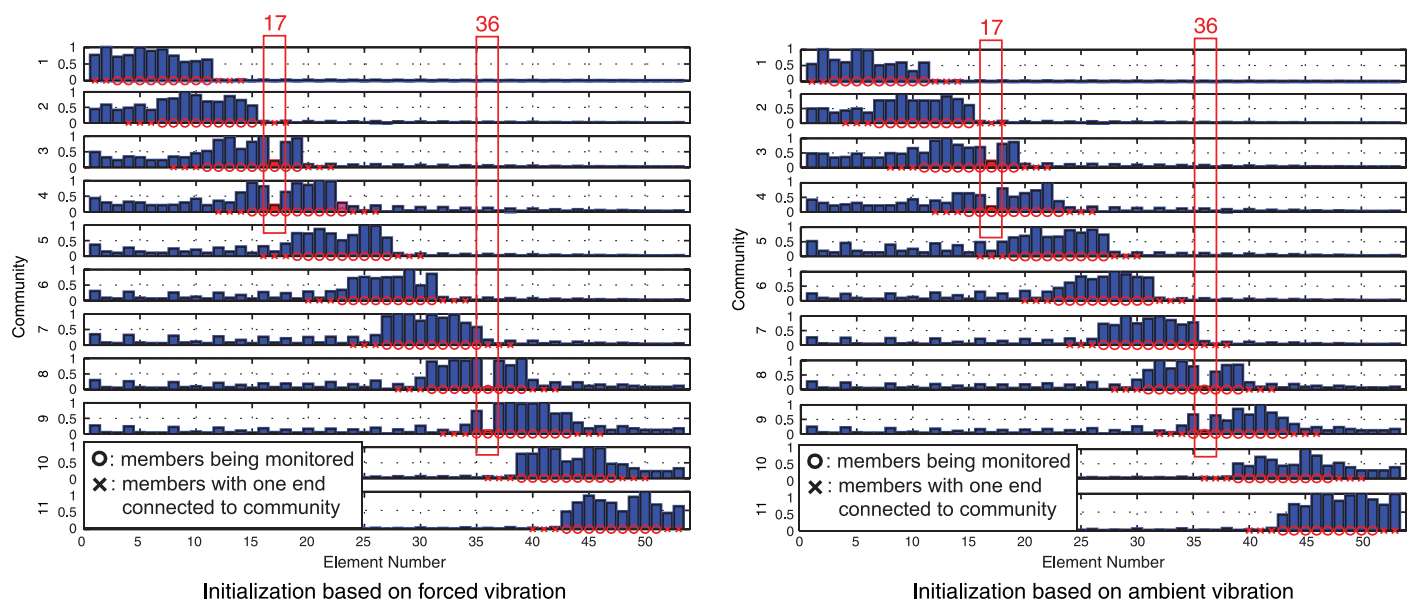

Figure 14. Normalized cumulative stress when elements 17 and 36 are damaged.

\section{CONCLUSIONS}

In this paper, a new DCS for SHM is proposed that is suitable for implementation on a network of densely distributed smart sensors. First, the basic concept of the DLV method was briefly introduced, and techniques for constructing flexibility matrix from both forced and ambient vibration were reviewed. An extension of the DLV method for continuous online SHM was then presented. Numerical studies using limited sensor information to detect damage were conducted that directly lead to the development of the DCS.

The proposed DCS approach differs from the traditional SHM algorithms because it does not rely on central acquisition and data processing. In the proposed approach, adjacent smart 


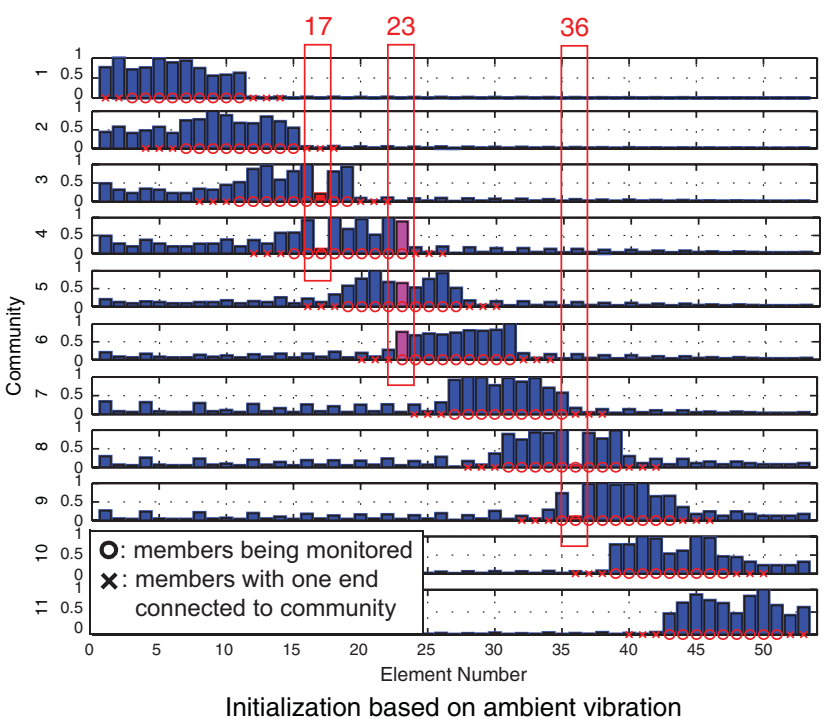

Figure. 15. Normalized cumulative stress when elements 17 and 36 have a $20 \%$ stiffness reduction (retaking data in communities, 4,5 , and 6 ).

sensors are grouped together to form sensor comminutes to monitor the local elements. Extraneous information is discarded before damage information is sent to the central station, therefore only limited information needs to be transferred wirelessly. Numerical simulations with noise included in measurements have been conducted with band-limited excitation. To better assess the performance of the proposed DCS approach, the magnitude and location of the excitations were changed before and after damage. Structural damage has been consistently identified using locally measured data from sensor communities. These results have shown the proposed DCS approach promising for continuous SHM with a densely distributed sensor network.

\section{ACKNOWLEDGEMENTS}

The authors gratefully acknowledge the support of the research by the National Science Foundation, under grant CMS 03-01140 (Dr S.C. Liu, Program Director).

\section{REFERENCES}

1. Doebling SW, Farrar CR, Prime MB, Shevitz DW. Damage identification and health monitoring of structural and mechanical systems from changes in their vibration characteristics: a literature review. Los Alamos National Laboratory Report LA-13070-MS, 1996.

2. Sohn H, Farrar CR, Hemez FM, Shunk DD, Stinemates DW, Nadler BR. A review of structural health monitoring literature: 1996-2001. Los Alamos National Laboratory Report LA-13976-MS, 2003.

3. Vandiver JK. Detection of structural failure on fixed platforms by measurement of dynamic response. Proceedings of the 7th Annual Offshore Technology Conference, 1975; 243-252. 
4. Cha PD, Tuck-Lee JP. Updating structural system parameters using frequency response data. Journal of Engineering and Mechanics 2000; 126(12):1240-1246.

5. West WM. Illustration of the use of modal assurance criterion to detect structural changes in an orbiter test specimen. Proceedings of Air Force Conference on Aircraft Structural Integrity, 1984; 1-6.

6. Fox CHJ. The location of defects in structures: a comparison of the use of natural frequency and mode shape data. Proceedings of the 10th International Modal Analysis Conference, Las Vegas, Nevada, vol. 1, 1992; 522-528.

7. Pandey AK, Biswas M. Damage detection in structures using changes in flexibility. Journal of Sound and Vibration 1994; 169(1):3-17.

8. Pandey AK, Biswas M. Damage diagnosis of truss structures by estimation of flexibility change. The International Journal of Analytical and Experimental Modal Analysis 1995; 10(2):104-117.

9. Bernal D. Load vectors for damage localization. Journal of Engineering and Mechanics 2002; 128(1):7-14.

10. Pandey AK, Biswas M, Samman MM. Damage detection from changes in curvature mode shapes. Journal of Sound and Vibration 1991; 145(2):321-332.

11. Salawu OS, Williams C. Damage location using vibration mode shapes. Proceedings of 12th International Modal Analysis Conference, 1994; 933-939.

12. Yao GC, Chang KC, Lee GC. Damage diagnosis of steel frames using vibrational signature analysis. Journal of Engineering and Mechanics 1992; 118(9):1946-1961.

13. Shin S, Hjelmstad KD. Damage detection and assessment of structural systems from measured response. Civil Engineering Studies 1994; SRS 593, UILU-ENG-94-2013, University of Illinois at Urbana-Champaign, IL.

14. Pothisiri T, Hjelmstad KD. Structural damage detection and assessment from modal response. Journal of Engineering and Mechanics 2003; 129(2):135-145.

15. Kudva J, Munir N, Tan P. Damage detection in smart structures using neural networks and finite element analysis. Proceedings of ADPA/AIAA/ASME/SPIE Conference on Active Materials and Adaptive Structures, 1991; 559-562.

16. Kirkegaard P, Nielsen S, Hansen H. Identification of nonlinear structures using recurrent neural networks. Proceedings of the 13th International Modal Analysis Conference, 1995; 1128-1134.

17. Spencer BF Jr, Ruiz-Sandoval M, Gao Y. Frontiers in structural health monitoring. Proceedings of China-Japan Workshop on Vibration Control and Health Monitoring of Structures and Third Chinese Symposium on Structural Vibration Control, Shanghai, China, 8-12 December, 2002.

18. Spencer BF Jr, Ruiz-Sandoval M, Krata N. Smart sensing technology: opportunities and challenges. Journal of Structural Control and Health Monitoring 2004; 11:349-368.

19. Gao Y, Spencer BF Jr, Bernal D. Experimental verification of the damage locating vector method. Journal of Engineering and Mechanics 2004, in review.

20. Gao Y, Spencer BF Jr. Online damage diagnosis of civil infrastructure employing a flexibility-based approach. Journal of Smart Materials and Structures 2005, in review.

21. Bernal D, Gunes B. Flexibility based approach for damage characterization: benchmark application. Journal of Engineering and Mechanics 2004; 130(1):61-70.

22. Juang JN, Pappa RS. An eigensystem realization algorithm for modal parameter identification and model reduction. Journal of Guidance Control and Dynamics 1985; 8:620-627.

23. Bernal D. Modal scaling from known mass perturbations. Journal of Engineering and Mechanics 2004; 130(9):10831088.

24. James GH III, Carne TG, Lauffer JP. The natural excitation technique (NExT) for modal parameter extraction from operating wind turbines. SAND92-1666, UC-261, Sandia National Laboratories, 1993. 